\title{
Dual-Rate Integration Using Partitioned Runge-Kutta Methods for Mechanical Systems with Interacting Subsystems ${ }^{\#}$
}

\author{
Siddhartha S. Shome, ${ }^{1, \dagger}$ Edward J. Haug, ${ }^{2, *}$ \\ and Laurent O. $\mathrm{Jay}^{3}$ \\ ${ }^{1}$ Software Engineer at Parametric Technology Corp. Simulation \\ Division, San Tomas Expy., Santa Clara, CA, USA \\ ${ }^{2}$ National Advanced Driving Simulator and Simulation Center, \\ The University of Iowa, Iowa City, IA, USA \\ ${ }^{3}$ Department of Mathematics, The University of Iowa, \\ Iowa City, IA, USA
}

\begin{abstract}
\#The work presented here was published in 2000 as Siddhartha S. Shome's Ph.D. dissertation at the University of Iowa, Dual-Rate Integration Using Partitioned Runge-Kutta Methods for Mechanical Systems with Interacting Subsystems.

${ }^{\dagger}$ Research work presented here done while a graduate student at the National Advanced Driving Simulator and Simulation Center at the University of Iowa. *Correspondence: Edward J. Haug, National Advanced Driving Simulator and Simulation Center, The University of Iowa, Iowa City, IA 52242, USA; E-mail: haug@nads-sc.uiowa.edu.
\end{abstract}

DOI: $10.1081 / \mathrm{LMBD}-200027930$

Copyright @ 2004 by Marcel Dekker, Inc.
1539-7734 (Print); 1539-7742 (Online) www.dekker.com 


\begin{abstract}
A framework is presented allowing dual-rate numerical integration of the equations of mechanical system dynamics to be considered as a form of Partitioned Runge-Kutta (PRK) integration. Certain coefficients of a PRK integrator are set to zero, so that Runge-Kutta integrators that constitute the PRK integrator can be made to have different numbers of stages. As a result, one Runge-Kutta integrator requires fewer function evaluations than the other does, which is a form of dual-rate integration. Well-established order of accuracy theory for PRK integrators is used to develop a rigorous methodology for designing explicit PRK dual-rate integrators. Stabilized Runge-Kutta theory developed for single-rate RungeKutta integrators is combined with PRK integrator theory to design PRK dual-rate integrators with the largest possible stability regions. Dual-rate PRK integrators created using these approaches are used to simulate the dynamics of vehicle systems that contain subsystems with higher frequency response characteristics than do the basic vehicle subsystems.
\end{abstract}

Key Words: Partitioned Runge-Kutta methods; Ordinary differential equations.

\title{
INTRODUCTION
}

Limitations of integrators that use the same step-size for numerically integrating all state variables of a system of ordinary differential equations (ODE) have been noted by researchers for some time. Since the late 1970s, attempts have been made to design numerical integrators that take into account high-frequency/low-frequency and stiff/nonstiff behavior of subsets of state variables in systems of ODE.

Most researchers have employed one of two basic approaches to solving this problem. The first approach, called multirate integration, is primarily applicable to systems in which state variables can be divided into high-frequency and low-frequency subsets. In this approach, stepsizes of integrators for different subsets of variables are different, while the integrator itself may be the same. For examples of this approach, see the work of Andrus (1979), Belytschko et al. (1979, 1993), Buzdugan (1999), Gear (1980, 1984), Gear and Wells (1984), Palusinski (1986), Solis (1996), and Wells (1982).

The second approach, called partitioned integration, is applied primarily to systems in which state variables can be partitioned into stiff 
and nonstiff subsets. Here, step-sizes of integrators for different subsets of variables are the same, but different types of integrators are used for different subsets. Examples of this approach include the work of Gunther and Rentrop (1994), Hofer (1976), Rentrop (1985), Strehmel and Weiner (1984), and Weiner et al. (1993). Another variation of this approach is discussed by Enright and Kamel (1979) and by Gear and Saad (1983).

This paper presents a method in which these two approaches are combined, using the framework of Partitioned Runge-Kutta integration. For two subsets of variables, two different Runge-Kutta integrators are used, one of which is a stabilized Runge-Kutta method.

In this paper, and in dual-rate integration in general, there are two fundamental assumptions:

(a) Linearized system eigenvalues corresponding to the two subsystems are widely separated. This is precisely the situation for which dual-rate integrators are designed. It is not an unreasonable assumption, based on the fact that two subsystems often represent physical phenomena that are very different from each other.

(b) For a given simulation time, there are more subsystem function evaluations for one subsystem compared to the other. It is assumed that the function evaluation that is done more often is significantly cheaper than function evaluation of the other subsystem.

\section{Partitioned Runge-Kutta View of Dual-Rate Integration}

Consider a set of ODE, partitioned as

$$
\left(\begin{array}{l}
y_{a} \\
y_{b}
\end{array}\right)^{\prime}=\left(\begin{array}{l}
f_{a}\left(y_{a}, y_{b}\right) \\
f_{b}\left(y_{a}, y_{b}\right)
\end{array}\right)
$$

The subsets $y_{a}$ and $y_{b}$ of variables are integrated numerically in a Partitioned Runge-Kutta (PRK) Method (Hairer et al., 1993):

$$
\begin{aligned}
k_{i} & =f_{a}\left(y_{a 0}+h \sum_{j=1}^{i-1} a_{i j} k_{j}, \quad y_{b 0}+h \sum_{j=1}^{i-1} \hat{a}_{i j} l_{j}\right) \\
l_{i} & =f_{b}\left(y_{a 0}+h \sum_{j=1}^{i-1} a_{i j} k_{j}, \quad y_{b 0}+h \sum_{j=1}^{i-1} \hat{a}_{i j} l_{j}\right) \\
y_{a 1} & =y_{a 0}+h \sum_{i=1}^{s} b_{i} k_{i}, \quad y_{b 1}=y_{b 0}+h \sum_{i=1}^{s} \hat{b}_{i} l_{i}
\end{aligned}
$$


Table 1. Butcher Tableaus depicting a PRK integration method.

\begin{tabular}{c|c|c|c|c|c}
\hline & $\hat{a}_{2,1}$ & & & & \\
\hline & $\hat{a}_{3,1}$ & $\hat{a}_{3,2}$ & & & \\
\hline & $\vdots$ & $\vdots$ & $\ddots$ & & \\
\hline & $\hat{a}_{s, 1}$ & $\hat{a}_{s, 2}$ & $\ldots$ & $\hat{a}_{s,(s-1)}$ & \\
\hline & $\hat{\boldsymbol{b}}_{1}$ & $\hat{\boldsymbol{b}}_{2}$ & $\ldots$ & $\ldots$ & $\hat{\boldsymbol{b}}_{s}$ \\
& $\hat{a}_{2,1}$ & & & & \\
\hline & $a_{2,1}$ & & & & \\
\hline & $a_{3,1}$ & $a_{3,2}$ & & & \\
\hline & $\vdots$ & $\vdots$ & $\ddots$ & & \\
\hline & $a_{s, 1}$ & $a_{s, 2}$ & $\ldots$ & $a_{s,(s-1)}$ & \\
\hline & $\boldsymbol{b}_{1}$ & $\boldsymbol{b}_{2}$ & $\ldots$ & $\ldots$ & $\boldsymbol{b}_{s}$ \\
\hline
\end{tabular}

where $s$ is the number of stages and coefficients $a_{i j}, b_{i}, \hat{a}_{i j}$, and $\hat{b}_{i}$ represent two different Runge-Kutta integrators.

It is customary to represent Runge-Kutta integrators by means of Butcher Tableaus. Since PRK integrators consist of two Runge-Kutta integrators, a PRK integrator is represented by two Butcher Tableaus, as shown in Table 1.

In all Runge-Kutta methods, the Taylor expansion of the solution generated by one step of numerical integration is set equal to the Taylor expansion of the exact solution, to a desired order. To do this, a theoretical framework has been developed for finding order conditions for Runge-Kutta integrators (Hairer et al., 1993) which, if satisfied by coefficients appearing in the Butcher Tableau, will ensure that integration is of the specified order of accuracy.

Order conditions have been developed for PRK integrators, using labeled P-trees (Hairer et al., 1993). For PRK integrators, each of the two Runge-Kutta integrators must satisfy order conditions imposed on all Runge-Kutta integrators. In addition, certain coupling conditions must be satisfied. Thus, more order conditions must be satisfied by PRK integrators than by nonpartitioned Runge-Kutta integrators of the same order. From the point of view of dual-rate integration, it is 
important to note that PRK integrators offer a rigorous theoretical framework in which a set of ODE can be partitioned and the resulting sets of variables can be discretized by different integration formulas.

One way of viewing dual-rate integration is to think of it as partitioning a set of ODE and using two different integration formulas (with different step-sizes) to discretize two subsets of state variables. Viewed from this perspective, when integrators comprising the dual-rate method are both Runge-Kutta integrators, the algorithm can be viewed as a special case of PRK integration.

The PRK approach to dual-rate integration is demonstrated using a linear example. Consider the system of linear ODE

$$
\left\{\begin{array}{l}
\dot{x} \\
\dot{y}
\end{array}\right\}=\left[\begin{array}{ll}
A & B \\
C & D
\end{array}\right]\left\{\begin{array}{l}
x \\
y
\end{array}\right\}
$$

where $x$ is the slow variable and $y$ is the fast variable that is to be integrated using a dual-rate method. As a test case, the forward Euler integrator with micro step-size $h / 3$ (for the fast variable $y$ ) and macro step-size $h$ (for the slow variable $x$ ) is considered.

The main challenge in dual-rate integration is to effectively account for the coupling between state variables that are integrated at different rates. For the current example, this is done in the following manner:

1) Influence of the slow variable on the fast variable. For slow first forward Euler integration over the larger step-size (macro step), linear interpolation of the slow variable is carried out as follows:

$$
\begin{aligned}
& x_{1}=x_{0}+h\left(A x_{0}+B y_{0}\right) \\
& x_{1 / 3}=\frac{2}{3} x_{0}+\frac{1}{3} x_{1}=x_{0}+\frac{h}{3}\left(A x_{0}+B y_{0}\right) \\
& x_{2 / 3}=\frac{1}{3} x_{0}+\frac{2}{3} x_{1}=x_{0}+\frac{2 h}{3}\left(A x_{0}+B y_{0}\right)
\end{aligned}
$$

2) Influence of the fast variable on the slow variable. Fast variable values at smaller step-size (micro steps) are ignored and the only fast variable values that are used for slow variable function evaluation are those at the beginnings of the macrosteps.

Using this approach, steps in dual-rate forward Euler integration are shown in Table 2. 
Table 2. One macro step and three micro steps in dual-rate forward Euler integration.

1. One slow step with step-size $h$
$x_{1}=x_{0}+h\left(A x_{0}+B y_{0}\right)$
2. $\quad$ Three fast steps with step-size $h / 3$
$y_{1 / 3}=y_{0}+\frac{h}{3}\left(C x_{0}+D y_{0}\right)$
$y_{2 / 3}=y_{1 / 3}+\frac{h}{3}\left[C\left(\frac{2}{3} x_{0}+\frac{1}{3} x_{1}\right)+D y_{1 / 3}\right]$
$y_{1}=y_{2 / 3}+\frac{h}{3}\left[C\left(\frac{1}{3} x_{0}+\frac{2}{3} x_{1}\right)+D y_{2 / 3}\right]$

\section{Dual-Rate Forward Euler as a PRK Integrator}

The algorithm of Table 2 can be written in the form of a PRK method, represented by two Butcher Tableaus in Table 3.

A valid interpretation of this PRK method is that it is not a multirate method at all, but a single-rate PRK method with step-size equal to $h$ and having three stages. However, since $b_{2}, b_{3}$, and $a_{32}$ are zero in the Butcher Tableau for the slow variable, only one stage variable is calculated for the slow variable, whereas three stage variables are calculated for the fast variable. Since the Butcher Tableaus of Table 3 represent the actual forward Euler method of Table 2, the fast stage variables at the three RungeKutta stages are just the values of the fast variable at the microsteps.

The theoretical framework of the PRK method can be utilized for dual-rate integration. By selecting some columns of the Butcher Tableau of one part of the PRK method to be zero, the number of function evaluations for the associated variable (the slow variable) can be made less than the number of function evaluations for the other variable (the fast variable). Within the theoretical framework of PRK methods, coefficients for the PRK method can be chosen such that properties of the integrator can be enhanced.

\section{Averaging and Interpolation in PRK Dual-Rate Integration}

In the forward Euler multirate integrator of Table 2, values of the fast variable at intermediate steps are ignored when evaluating functions of the slow variable. Only the fast variable value at the beginning of 
Table 3. Butcher Tableaus representing conventional forward Euler dual-rate.

\begin{tabular}{l|c|c|c}
$\mathbf{1} / \mathbf{3}$ & $1 / 3$ & & \\
\hline $\mathbf{2} / \mathbf{3}$ & $2 / 3$ & 0 & \\
\hline & $\mathbf{1}$ & $\mathbf{0}$ & $\mathbf{0}$
\end{tabular}

\begin{tabular}{l|l|l|l}
$\mathbf{1} / \mathbf{3}$ & $1 / 3$ & & \\
\hline $\mathbf{2} / \mathbf{3}$ & $1 / 3$ & $1 / 3$ & \\
\hline & $\mathbf{1} / \mathbf{3}$ & $\mathbf{1} / \mathbf{3}$ & $\mathbf{1} / \mathbf{3}$
\end{tabular}

the macro time step is used for this function evaluation. This means that the intermediate fast variable values are neglected when slow variable functions are evaluated; i.e., in the example of Table 2, $y_{1 / 3}$ and $y_{2 / 3}$ are neglected and only $y_{0}$ is used in calculating $x_{1}$. The PRK Dual Rate method can be viewed as a way to take a weighted average of the fast variable stages, while at the same time maintaining the order of integration; i.e., in the example of Table 2 , instead of just using $y_{0}$, a weighted average of $y_{0}, y_{1 / 3}$, and $y_{2 / 3}$ can be used to calculate $x_{1}$.

Consider a PRK Dual Rate method with three stages for the fast variable and one stage for the slow variable, using Butcher Tableaus from Table 4. The resulting PRK formulas for the linear ODE of Eq. (3) are

$$
\begin{aligned}
& l_{1}=C x_{0}+D y_{0} \\
& k_{2}=A x_{0}+B\left(y_{0}+h a_{21} l_{1}\right) \\
& l_{2}=C x_{0}+D\left(y_{0}+h a_{21} l_{1}\right) \\
& l_{3}=C\left(x_{0}+h \hat{a}_{32} k_{2}\right)+D\left(y_{0}+h a_{31} l_{1}+h a_{32} l_{2}\right) \\
& x_{1}=x_{0}+h k_{2} \\
& y_{1}=y_{0}+h\left(b_{1} l_{1}+b_{2} l_{2}+b_{3} l_{3}\right)
\end{aligned}
$$

The major difference between the PRK method of Table 4 and the one in Table 3 is that, in the PRK of Table $3, k_{1}$ is used and $k_{2}$ and $k_{3}$ are not used. On the other hand, for the PRK of Table $4, k_{2}$ is used and $k_{1}$ 
Table 4. Butcher Tableaus representing partitioned Runge-Kutta dual-rate.

\begin{tabular}{|c|c|c|c|}
\hline $1 / 3$ & 0 & & \\
\hline \multirow[t]{2}{*}{$2 / 3$} & 0 & $\hat{a}_{32}$ & \\
\hline & $\mathbf{0}$ & 1 & 0 \\
\hline $1 / 3$ & $a_{21}$ & & \\
\hline \multirow[t]{2}{*}{$2 / 3$} & $a_{31}$ & $a_{32}$ & \\
\hline & $\mathbf{b}_{1}$ & $\mathbf{b}_{2}$ & $\mathbf{b}_{3}$ \\
\hline
\end{tabular}

and $k_{3}$ are not required. This means that the slow function [which, in the case of Eq. (3), is $A x+B y$ ] is not evaluated using just the values of the state variables at the beginning of the macrostep. Instead, one or more fast variable stages are evaluated (analogous to microsteps) using the value of the slow variable at the beginning of the macrostep. A linear combination of these fast variable stages (analogous to weighted averaging) is used to evaluate the slow variable function. This linear combination is taken over the current macrostep. In other words, the slow variable function evaluation [Eq. (8)] uses not just the fast variable value at the beginning of the macrostep $\left(y_{0}\right)$, but a linear combination of $y_{0}$ and a fast variable stage value $\left(l_{1}\right)$. Moreover, interpolation of the slow variable, which is required in conventional multirate methods to evaluate the fast variable function, is accomplished by the integration algorithm (here by the coefficient $\hat{a}_{32}$ ), and no additional interpolation algorithm is required.

\section{Design of PRK Dual-Rate Integrators}

A PRK dual-rate integrator consists of two coupled Runge-Kutta integrators, each having a different number of stages. The Runge-Kutta integrator that is used to integrate slow variables has a smaller number of stages than the Runge-Kutta integrator that is used to integrate fast variables. The order of the slow variable integrator is limited by the following theorem (Hairer et al., 1993):

Theorem 1. An $s$-stage explicit Runge-Kutta method cannot have order greater than $s$. 
Note that Theorem 1 is for single-rate Runge-Kutta integrators only.

Since the fast variable integrator has a larger number of stages than the slow variable integrator, it may be expected that the order of accuracy of the fast variable may be higher than the order of accuracy of the slow variable. Indeed it is possible that local error in fast variable integration can be one order higher than local error in slow variable integration. However, in the general case, due to coupling between fast and slow variables, an error in slow variable integration is transmitted to the fast variable, and the order of accuracy is the same for both variables. Thus, in the general case, the order of a PRK dual-rate integrator is limited by the number of slow variable stages. This is illustrated by a simple example in Appendix A.

In numerical integration, step-sizes are usually limited by two requirements: (1) truncation error must remain below a certain limit, and (2) numerical stability must be maintained. For the intended application of real-time vehicle system simulation, the necessity of maintaining numerical stability is usually more critical than the necessity of keeping the truncation error below a given limit. Keeping this in mind, efforts have been made to design PRK dual-rate integrators with enhanced stability. For other applications, PRK dual-rate integrators may be designed for increased accuracy, within limits established by Theorem 1.

Stability region Approach for Designing PRK Dual-Rate Integrators

For single rate integration, stability analysis is carried out using the Dahlquist test equation,

$$
\dot{x}=\lambda x
$$

An $s$-stage explicit Runge-Kutta method applied to this test problem yields, for the $(m+1)$ th step (Hairer and Wanner, 1996),

$$
x_{m+1}=R(h \lambda) x_{m}
$$

where

$$
R(z)=1+z \sum_{j} b_{j}+z^{2} \sum_{j, k} b_{j} a_{j k}+z^{3} \sum_{j, k, l} b_{j} a_{j k} a_{k l}+\cdots
$$


is a polynomial of degree $\leq s, z=h \lambda$, and $a_{j i}$ and $b_{j}$ are the coefficients of the Runge-Kutta method. The stability domain of a Runge-Kutta method is

$$
\mathbf{S}=\{z \in C ;|R(z)| \leq 1\}
$$

For a given Runge-Kutta integrator and a complex value of $z, R(z)$ is a complex number, so the stability region $\mathbf{S}$ is in two-dimensional space.

The scalar test equation of Eq. (13) is not sufficient for analyzing the stability of PRK dual-rate integrators (or PRK integrators in general), because in PRK integrators, two different integration techniques are applied to two different sets of variables. The simplest equation that can be used to study the stability of PRK dual-rate methods is a modified form of the Dahlquist equation,

$$
\left[\begin{array}{l}
\dot{x} \\
\dot{y}
\end{array}\right]=\left[\begin{array}{ll}
\lambda_{s s} & \lambda_{s f} \\
\lambda_{f s} & \lambda_{f f}
\end{array}\right]\left[\begin{array}{l}
x \\
y
\end{array}\right]
$$

or, in matrix form,

$$
\dot{\mathbf{q}}=\Lambda \mathbf{q}
$$

Consider a PRK integrator defined by the Butcher Tableaus shown in Table 1, applied to the ODE of Eq. (17). The first step in numerical integration is

$$
\begin{aligned}
& k_{i}=\lambda_{s s}\left(x_{0}+h \sum_{j=1}^{s} a_{i j} k_{j}\right)+\lambda_{s f}\left(y_{0}+h \sum_{j=1}^{s} \hat{a}_{i j} l_{j}\right) \\
& l_{i}=\lambda_{f s}\left(x_{0}+h \sum_{j=1}^{s} a_{i j} k_{j}\right)+\lambda_{f f}\left(y_{0}+h \sum_{j=1}^{s} \hat{a}_{i j} l_{j}\right) \\
& x_{1}=x_{0}+h \sum_{i=1}^{s} b_{i} k_{i} \\
& y_{1}=y_{0}+h \sum_{i=1}^{s} \hat{b}_{i} l_{i}
\end{aligned}
$$

Defining

$$
\begin{aligned}
& \mathbf{q}_{i}=\left[\begin{array}{c}
x_{i} \\
y_{i}
\end{array}\right] ; \quad \mathbf{p}_{i}=\left[\begin{array}{c}
k_{i} \\
l_{i}
\end{array}\right] ; \quad \mathbf{A}_{i j}=\left[\begin{array}{cc}
a_{i j} & 0 \\
0 & \hat{a}_{i j}
\end{array}\right] ; \quad \mathbf{B}_{i}=\left[\begin{array}{cc}
b_{i} & 0 \\
0 & \hat{b}_{i}
\end{array}\right] \\
& \mathbf{Z}=\left[\begin{array}{ll}
z_{s s} & z_{s f} \\
z_{f s} & z_{f f}
\end{array}\right]=h \Lambda=\left[\begin{array}{ll}
h \lambda_{s s} & h \lambda_{s f} \\
h \lambda_{f s} & h \lambda_{f f}
\end{array}\right]
\end{aligned}
$$


and

$$
\mathbf{R}_{i}=\mathbf{Z}+\mathbf{Z} \sum_{j=1}^{s} \mathbf{A}_{i j} \mathbf{R}_{j} \quad \text { and } \quad \mathbf{S}=\mathbf{I}+\sum_{i=1}^{s} \mathbf{B}_{i} \mathbf{R}_{i}
$$

Eqs. (20) and (21) can be written as

$$
\mathbf{q}_{1}=\mathbf{S q}_{0}
$$

The matrix $\mathbf{S}$ of Eq. (22) is called the stability matrix of the PRK method. For stability analysis, properties of this matrix must be investigated. For the PRK integration method to be stable, the magnitude of the largest eigenvalue of $\mathbf{S}$ must be less than one. If $\mathbf{A}_{i j}$ and $\mathbf{B}_{i}$ are known, $\mathbf{S}$ can be expressed as a function of $z_{s s}, z_{s f}, z_{f s}$, and $z_{f f}$. Eigenvalues of $\mathbf{S}$ can be found by solving the characteristic equation

$$
|\mathbf{S}-\lambda \mathbf{I}|=0
$$

for $\lambda$. Integration is stable if the magnitudes of both eigenvalues are less than 1 ; i.e.,

$$
\left|\lambda_{1}\left(z_{s s}, z_{s f}, z_{f s}, z_{f f}\right)\right|<1 \text { and }\left|\lambda_{2}\left(z_{s s}, z_{s f}, z_{f s}, z_{f f}\right)\right|<1
$$

Equation (24) defines the stability region of the PRK dual-rate integration method. Since there are four complex variables, as opposed to one complex variable for single rate integration methods, the stability region is in eight-dimensional space. Unfortunately a stability region in eight-dimensional space is not very intuitive and cannot be directly used to select coefficients for PRK dual-rate integrators.

\section{Decoupled Stability Approach for Enhanced Stability of PRK} Dual-Rate Integrators

A comprehensive stability theory for PRK integrators does not exist. However, a substantial amount of work has been done in developing a stability theory for single-rate Runge-Kutta integrators. In the decoupled stability approach, the idea is to neglect the effect of coupling on stability and design the Runge-Kutta integrators that make up the PRK dual-rate integrator in such a way that the individual stability regions of the two integrators (ignoring coupling) are the largest possible for the given numbers of stages. It should be noted that, even though the influence of coupling on stability is neglected, coupling is still considered for the order 
conditions; and all required order conditions are satisfied. Thus, the order of accuracy of numerical integration is maintained.

For most PRK dual-rate integrators, the order of integration for slow variables is equal to the number of slow variable stages. For such an integrator, the stability region of the slow integrator is fixed and cannot be changed by changing integrator coefficients. In the decoupled stability approach, the idea is to maximize the stability region of the fast integrator.

\section{Stabilized Runge-Kutta Methods}

It is often desirable to have explicit Runge-Kutta integrators that have large stability regions. One way of increasing the stability regions of Runge-Kutta integrators is to construct explicit Runge-Kutta integrators that have larger numbers of stages than the minimum required to satisfy integration order conditions and to select integrator coefficients in a way that enlarges the stability region. A number of researchers have worked on developing rigorous methodologies for doing this; e.g., Kinnmark and Gray (1984a, b), Sommeijer et al. (1998), van der Houwen (1977), and Verwer (1996).

As shown earlier, for the scalar Dahlquist test equation of Eq. (13), one step of $s$-stage Runge-Kutta integration is, from Eq. (14),

$$
x_{m+1}=R(z) x_{m}
$$

where $R(z)$ is a polynomial of degree $\leq s$. For an $s$-stage, $p$-order RungeKutta method where $s>p$, the stability function is

$$
R(z)=1+z+\frac{z^{2}}{2 !}+\frac{z^{3}}{3 !} \cdots+\frac{z^{p}}{p !}+\beta_{p+1} z^{p+1}+\beta_{p+2} z^{p+2}+\cdots+\beta_{s} z^{s}
$$

Researchers have tried to find, for $s$ and $p$ with $s>p$, coefficients $\beta_{p+1}, \beta_{p+2}, \ldots, \beta_{s}$ for a polynomial of the form of Eq. (25), such that the corresponding stability domain is as large as possible in the desired direction (either negative real axis or imaginary axis). After finding coefficients $\beta_{p+1}, \beta_{p+2}, \ldots, \beta_{s}$ that maximize the stability region along the desired axis, coefficients of the Runge-Kutta method are chosen so that the stability function of the Runge-Kutta integrator is the same as the desired stability function. Such integrators are called Stabilized RungeKutta integrators. 
Table 5. Coefficients of Eq. (25) for maximum stability along an imaginary axis.

\begin{tabular}{cccccc}
\hline$s$ & $\beta_{3}$ & $\beta_{4}$ & $\beta_{5}$ & $\beta_{6}$ & $\beta_{7}$ \\
\hline 3 & $1 / 4$ & & & & \\
5 & $3 / 16$ & $1 / 32$ & $1 / 128$ & & \\
7 & $19 / 108$ & $1 / 27$ & $2 / 243$ & $1 / 1458$ & $1 / 8748$ \\
\hline
\end{tabular}

Most work on stabilized Runge-Kutta methods has been motivated by the desire to integrate equations for which the real parts of the eigenvalues of the Jacobian are large. Most of that work focuses on solving parabolic partial differential equations. However, for simulation of vehicle systems with stiff tire models, which is the target application for this research, stabilized Runge-Kutta integrators that have large stability regions along the imaginary axis are of greatest interest.

Polynomials for maximizing stability regions of Runge-Kutta integrators along the imaginary axis have been derived by van der Houwen (1977) and by Kinnmark and Gray (1984a, 1984b). Polynomials that maximize the stability region along the imaginary axis for first order integration are also presented by Hairer and Wanner (1996). van der Houwen (1977) derived polynomials of degree $s>2$ that match Taylor expansion coefficients up to second order and give the largest possible stability region along the imaginary axis. van der Houwen (1977) and Kinnmark and Gray (1984a) developed such polynomials for odd numbers of stages.

For $s$-stage second order explicit Runge-Kutta integration, coefficients $\beta_{3}, \beta_{4}, \ldots \beta_{s}$ of Eq. (25), which give the maximum possible stability region along the imaginary axis, for integrators up to seven stages, are given in Table 5 (Kinnmark and Gray, 1984a; van der Houwen, 1977).

Kinnmark and Gray (1984b) developed maximum imaginary stability region polynomials that match Taylor expansion up to third order. They have also developed, for even numbers of stages, maximum imaginary stability region polynomials that match Taylor expansion up to fourth order.

Stabilized Runge-Kutta Theory Applied to PRK Dual-Rate Design

In an explicit PRK dual-rate integrator, there are two coupled Runge-Kutta integrators that have different numbers of stages. The order of integration for both Runge-Kutta integrators is limited by the number 
of stages of the integrator having the smallest number of stages, since the two integrators are coupled. The integrator with the largest number of stages has $s$ stages, but is $p$ order accurate, with $s>p$, where $s$ is the number of stages of the fast variable integrator and $p$ is the number of nonzero columns of the slow variable integrator and the order of the method. The fast variable integrator, therefore, can be designed using stabilized Runge-Kutta theory. This is a decoupled stability approach, which means that, while designing PRK dual-rate integrators, coupling between the two integrators is neglected, as far as the effect on stability is concerned. However, all PRK order conditions, including coupling order conditions up to the desired order $p$, are satisfied.

As an example, consider a PRK dual-rate integrator that has two stages for the slow integrator and five stages for the fast integrator. The coefficients of this $2-5$ PRK dual rate integrator are chosen such that the six order conditions that are required for second order integration, are satisfied. Since up to second order conditions are satisfied, the individual stability functions of the slow and the fast Runge-Kutta integrators are (Hairer et al., 1993)

$$
\begin{aligned}
R_{2-\text { stg }}^{\text {slow }}(z)= & 1+z+\frac{1}{2} z^{2} \\
R_{5 \text {-stg }}^{\mathrm{fast}}(z)= & 1+z+\frac{1}{2} z^{2}+z^{3} \sum_{j, k, l} b_{j} a_{j k} a_{k l}+z^{4} \sum_{j, k, l, m} b_{j} a_{j k} a_{k l} a_{l m} \\
& +z^{5} \sum_{j, k, l, m, p} b_{j} a_{j k} a_{k l} a_{l m} a_{m p}
\end{aligned}
$$

The 2-5 PRK dual-rate integrator coefficients can be chosen so that the stability function of the five-stage part of the 2-5 PRK dual-rate integrator is the same as the stability function that gives the largest possible stability region along the imaginary axis, as shown in Eq. (25) and Table 5. In other words, a 2-5 PRK dual-rate integrator is designed that satisfies all conditions, including coupling order conditions, for second order integration and the following additional conditions for the five-stage part of the 2-5 PRK dual-rate integrator:

$$
\begin{aligned}
& \sum_{j, k, l} b_{j} a_{j k} a_{k l}=\frac{3}{16} \\
& \sum_{j, k, l, m} b_{j} a_{j k} a_{k l} a_{l m}=\frac{1}{32} \\
& \sum_{j, k, l, m, p} b_{j} a_{j k} a_{k l} a_{l m} a_{m p}=\frac{1}{128}
\end{aligned}
$$


Table 6. Coefficients of the $2-5$ PRK dual-rate integrator.

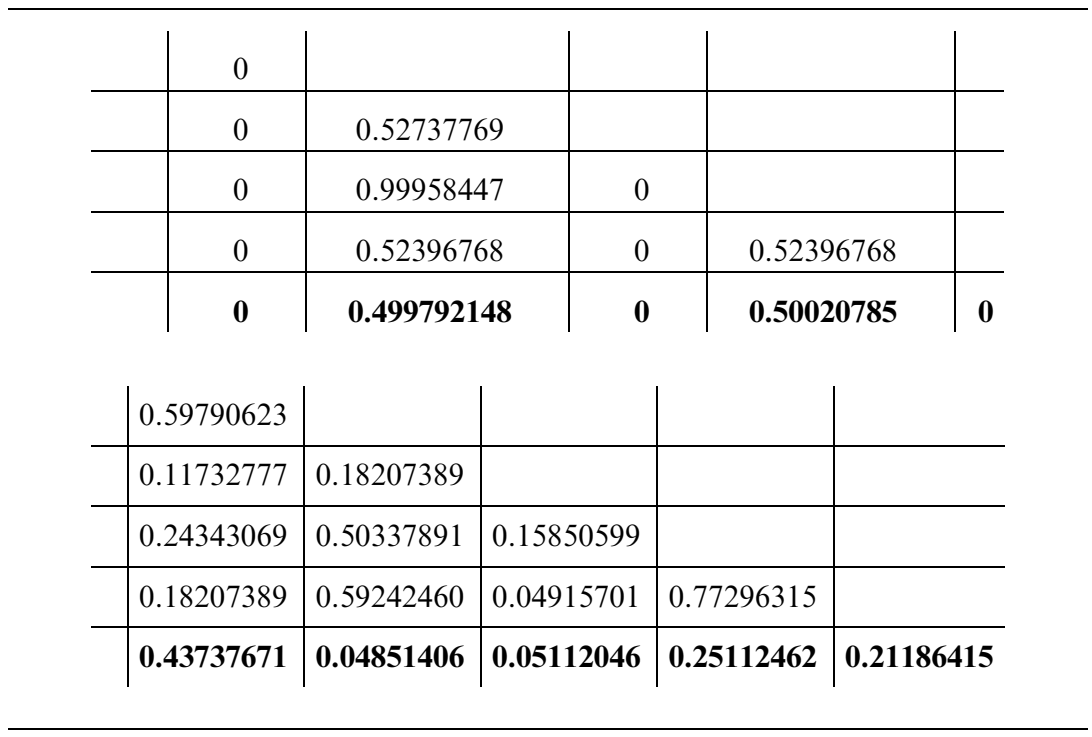

A 2-5 PRK dual-rate integrator designed using this method is shown in Table 6. Stability regions of the slow and the fast integrators are shown in Fig. 1.

\section{Vehicle Examples}

Consider dynamic simulation using numerical integration for the planar quarter car models shown in Fig. 2. In model (A), a spring and a damper representing the suspension and the tire, act between the chassis body and ground. In model (B), there are two masses: (1) the unsprung mass representing the wheel and (2) the sprung mass representing the chassis. The suspension is represented by a spring and damper, and the tire is modeled as a spring. In model $(\mathrm{C})$, the chassis and suspension models remain the same as in model (B), but the tire model is changed from a spring to a more complex rigid ring tire model. A detailed description of the planar rigid ring tire model can be found in Appendix B.

A second order, two-stage Runge-Kutta integrator (RK2) is used for simulation. For each of the three models, a number of step-sizes are used 


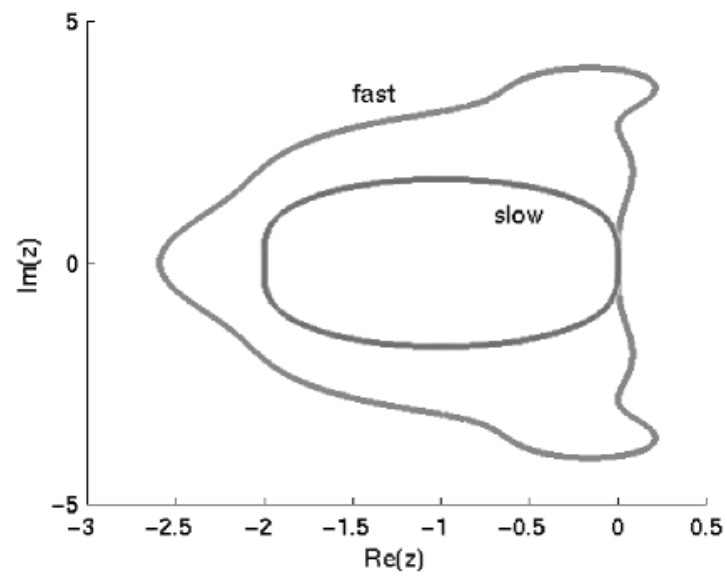

Figure 1. Stability regions of 2-5 PRK dual-rate using stabilized Runge-Kutta. (View image in color online.)
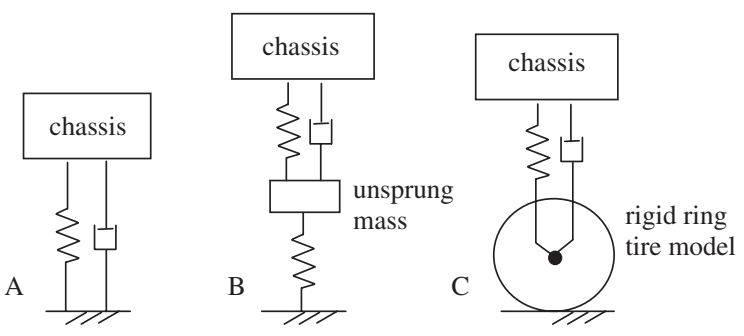

Figure 2. Planar quarter car models.

for numerical integration, and the largest step-size that can be taken with the RK2 integrator, while maintaining stability, is recorded in Table 7.

The largest step-size that can be taken by the integrator varies significantly, depending on which of the three models is being simulated. When the rigid ring tire model is included in the vehicle system model, the largest step-size that can be taken by the second order, two-stage Runge-Kutta integrator (RK2) is much smaller than the largest stepsize that can be used for a vehicle model without a rigid ring tire model. This example suggests that it is the rigid ring tire model that is forcing the integrator to take a small step-size. This opens up the possibility of improving computational efficiency by using PRK dual-rate integrators and integrating the tire variables using a Runge-Kutta integrator with a 
Table 7. Step-size requirements for stability for planar quarter car model.

\begin{tabular}{ccc}
\hline & Model & $\begin{array}{c}\text { Maximum Step-Size for } \\
\text { Stable Numerical Integration } \\
\text { (2-stage Runge-Kutta, RK2) }\end{array}$ \\
\hline A $\quad \begin{array}{l}\text { Chassis, one spring and one damper } \\
\text { B } \quad \begin{array}{c}\text { Chassis (sprung mass) and wheel } \\
\text { (unsprung mass), spring and } \\
\text { damper suspension, spring tire }\end{array}\end{array}$ \\
C Chassis, spring and damper suspension, \\
rigid ring tire model
\end{tabular}

larger number of stages than the Runge-Kutta integrator used to integrate vehicle variables. Use of PRK dual-rate integration is most promising when slow variable functions are much more expensive to evaluate than fast variable functions. This is the case for spatial multibody models of vehicles interacting with subsystems.

Planar Vehicle Model with Planar Rigid Ring Tire Model

PRK dual-rate integration is carried out for the planar vehicle model shown in Fig. 3. Twenty-eight ODE represent this planar vehicle model, with planar rigid ring tires. The 28 state variables are partitioned into 14 fast variables and 14 slow variables. The fast variables correspond to the positions and velocities of the two rigid ring bodies and tire slips for the two tires. The slow variables correspond to independent positions and velocities of the two wheel hub bodies and the chassis body.

The coupled fast and slow ODE can be written as

$$
\dot{\mathbf{y}}=\Pi(\mathbf{y}, \mathbf{x})
$$

and

$$
\dot{\mathbf{x}}=\Gamma(\mathbf{y}, \mathbf{x})
$$

where $\mathbf{x}$ is the vector of fast variables and $\mathbf{y}$ is the vector of slow variables.

The Jacobian of these equations can be constructed using numerical differentiation. The Jacobian is partitioned into four sub-Jacobians,

$$
\mathbf{J}=\left[\begin{array}{ll}
\mathbf{J}_{s s} & \mathbf{J}_{s f} \\
\mathbf{J}_{f s} & \mathbf{J}_{f f}
\end{array}\right]=\left[\begin{array}{ll}
\frac{\partial \Pi(\mathbf{y}, \mathbf{x})}{\partial \mathbf{y}} & \frac{\partial \Pi(\mathbf{y}, \mathbf{x})}{\partial \mathbf{x}} \\
\frac{\partial \Gamma(\mathbf{y}, \mathbf{x})}{\partial \mathbf{y}} & \frac{\partial \Gamma(\mathbf{y}, \mathbf{x})}{\partial \mathbf{x}}
\end{array}\right]
$$




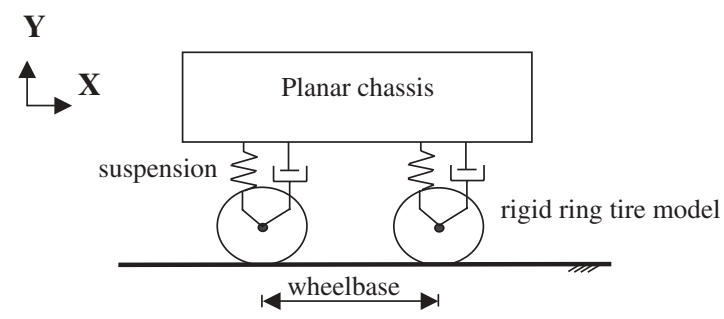

Figure 3. Planar vehicle model.

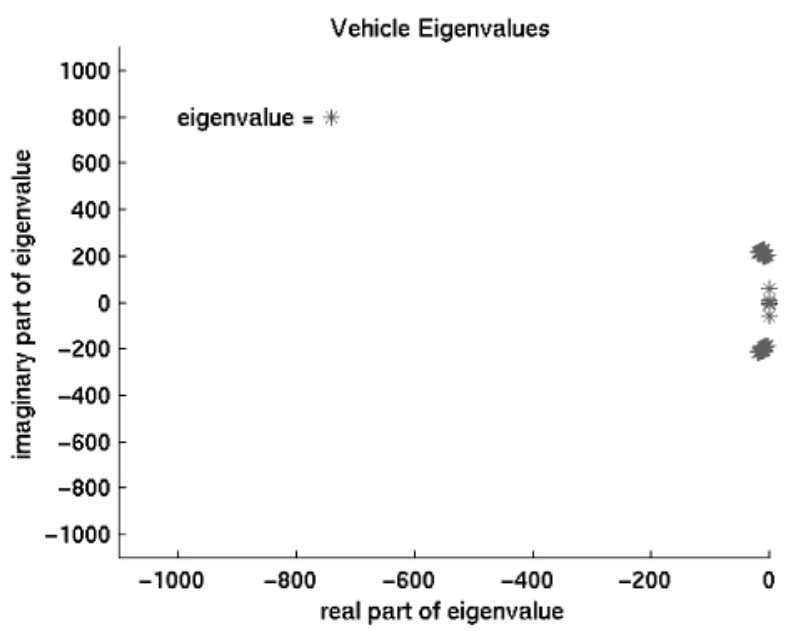

Figure 4. Distribution of slow (vehicle) eigenvalues for planar vehicle model. (View image in color online.)

The sub-Jacobian $\mathbf{J}_{s s}$ represents the influence of the slow state variables on the slow equations and $\mathbf{J}_{s f}$ represents the influence of the fast state variables on the slow equations. The sub-Jacobian $\mathbf{J}_{f f}$ represents the influence of the fast state variables on the fast equations and $\mathbf{J}_{f s}$ represents the influence of the slow state variables on the fast equations. A $50 \mathrm{~s} \mathrm{simulation} \mathrm{is} \mathrm{run,} \mathrm{with} \mathrm{torques} \mathrm{applied} \mathrm{to} \mathrm{both} \mathrm{wheels} \mathrm{of} \mathrm{the} \mathrm{planar}$ vehicle model. Identical torques are applied to each wheel.

Eigenvalues of $\mathbf{J}_{s s}$ and $\mathbf{J}_{f f}$ during this simulation run are plotted in Figs. 4 and 5, respectively. As can be seen, fast subsystem eigenvalues are much larger in magnitude than are slow subsystem eigenvalues. It can also be seen that for the fast subsystem eigenvalues, imaginary parts are much larger in magnitude than real parts. 


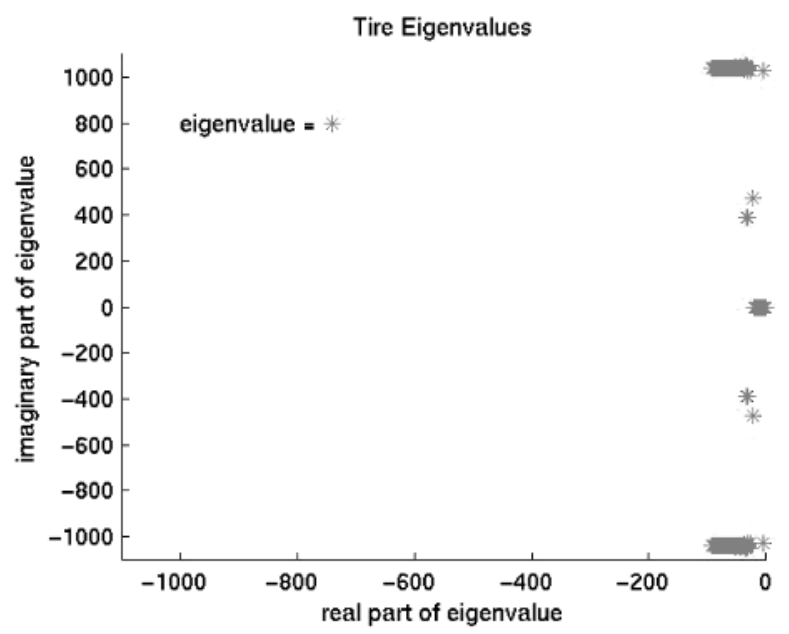

Figure 5. Distribution of fast (tire) eigenvalues for planar vehicle model. (View image in color online.)

Table 8. Increased step-size with 2-5 PRK integrator for planar vehicle model.

\begin{tabular}{lc} 
Integrator & Max. Step-Size for Stability \\
\hline RK2 (Single-Rate) & $0.0005 \mathrm{~s}$ \\
2-5 PRK (Dual-Rate) & $0.002 \mathrm{~s}$ \\
\hline
\end{tabular}

The 2-5 PRK dual-rate integrator shown in Table 6 is used to carry out integration of the planar vehicle model with the planar rigid ring tire model whose fast and slow eigenvalues are shown in Figs. 4 and 5. The two-stage part of the PRK integrator is used for the slow variables and the five-stage part of the PRK integrator is used for the fast variables. Table 8 shows computational performance of the 2-5 PRK dual-rate integrator of Table 6 and compares it with the performance of a single-rate, second order Runge-Kutta integrator.

Spatial Vehicle Model with Spatial Rigid Ring Tire Model

Simulation is carried out for a spatial model of the U.S. Army High Mobility Multipurpose Wheeled Vehicle (HMMWV). A multibody dynamics model consisting of 10 bodies is used, as described by 


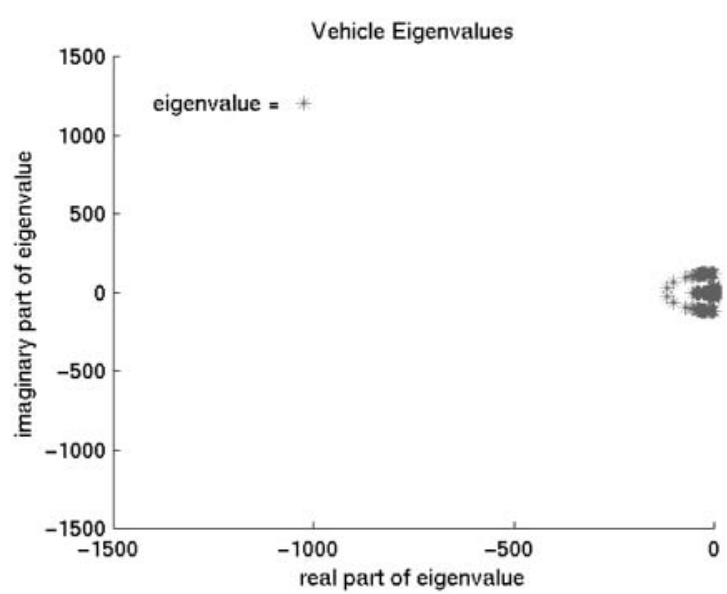

Figure 6. Slow (vehicle) eigenvalue distribution: HMMWV with rigid ring tire. (View image in color online.)

Serban et al. (1998). The vehicle tires are modeled as spatial rigid ring tires, as described by Maurice et al. (1997). State variables corresponding to the multibody vehicle model are taken as the slow variables, while state variables corresponding to the rigid ring tire model and the powertrain are taken as fast variables.

As in the planar vehicle model example, the Jacobian is constructed numerically and is partitioned into fast and slow sub-Jacobians. Eigenvalues of the slow and fast sub-Jacobians are plotted in Figs. 6 and 7, respectively. As can be seen, the eigenvalue distribution is very similar to the eigenvalue distribution for the planar vehicle model with planar rigid ring tires shown in Figs. 4 and 5. Table 9 shows computational performance of the 2-5 PRK dual-rate integrator of Table 6, and compares it with the performance of a single-rate, second order RungeKutta integrator. As can be seen, the 2-5 PRK dual-rate integrator is able to take a step-size that is three times as large as the single-rate integrator. This translates into a reduction in CPU time by a factor of 2.4. The CPU times shown in Table 9 are obtained on an SGI Onyx computer on a single R10000 processor.

Integrators with Larger Stability Regions

An effort was made to design PRK dual-rate integrators with higher dual-rate ratios. Dual-rate integrators including 2-6, 2-7, 2-8, and 2-9 


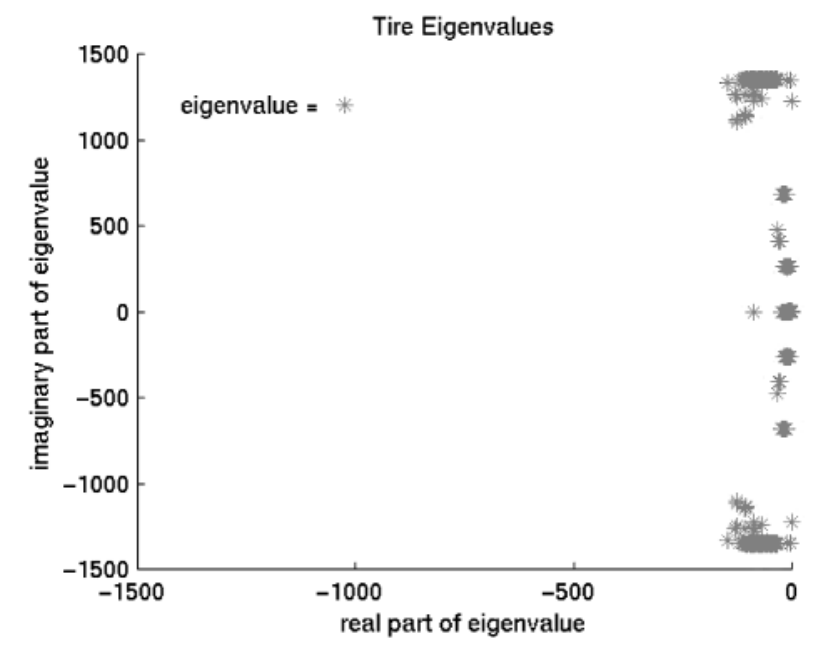

Figure 7. Fast (tire) eigenvalue distribution: HMMWV with rigid ring tire. (View image in color online.)

Table 9. Spatial HMMWV with tire results using 2-5 PRK dual-rate integrator.

\begin{tabular}{lcc}
\hline Integrator & Max. Step-Size for Stability & CPU Time \\
\hline RK2 (Single-Rate) & $0.001 \mathrm{~s}$ & $382.08 \mathrm{~s}$ \\
2-5 PRK (Dual-Rate) & $0.003 \mathrm{~s}$ & $162.74 \mathrm{~s}$ \\
\hline
\end{tabular}

PRK have been designed using stabilized Runge-Kutta theory. However, it was found that while the stability properties of the integrators allow them to take larger step-sizes, truncation errors are so large that results obtained are very different from the actual solutions.

For example, consider a 2-6 PRK dual-rate integrator. This integrator has been designed such that the six-stage integrator that forms part of the PRK dual-rate integrator has a larger stability region along the imaginary axis than does the five-stage integrator in the 2-5 PRK dual-rate integrator of Table 6.

If the planar vehicle model with planar rigid ring tires is simulated using the 2-6 PRK dual-rate integrator, a step-size of $0.0025 \mathrm{~s}$ can be taken, compared to the maximum step-size of $0.002 \mathrm{~s}$ of the 2-5 PRK dual-rate integrator of Table 6. However, it is seen from Fig. 8 that with this large step-size truncation error is quite large. For a simulation time 


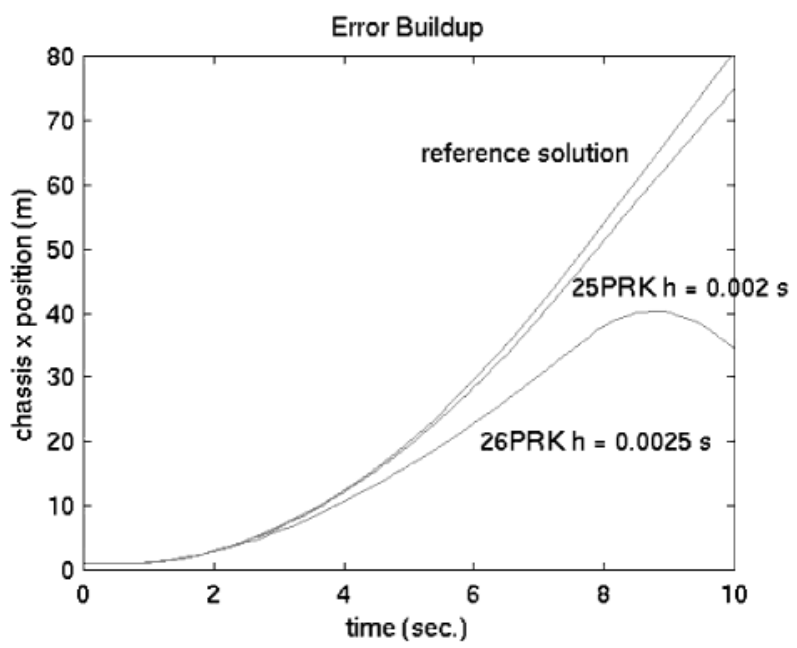

Figure 8. Truncation error buildup for 2-5 and 2-6 PRK dual-rate integrators. (View image in color online.)

of $10 \mathrm{~s}$, truncation error is so large that the solution is qualitatively different from the reference solution. The reference solution is a solution that is obtained by integrating the same differential equations using a fourth-order Runge-Kutta integrator with a constant step size of $10^{-9}$. These results indicate that for second-order integration, the dual-rate ratio cannot be usefully increased much above $2: 5$. However, this also gives a very encouraging indication that if PRK dual-rate integrators are designed to have a higher order of accuracy, which is within the realm of possibility, the computational speedup achieved can be even greater than what is shown in this paper.

\section{CONCLUSIONS AND RECOMMENDATIONS}

A new framework for dual-rate integration in which dual-rate integration is considered to be a special case of PRK integration has been developed. By doing this, the rigorous theoretical framework that exists for PRK integration can be utilized for dual-rate integration. The new framework allows integrators to be specifically designed for dual-rate integration, instead of the earlier practice of modifying singlerate integrators to serve as dual-rate integrators. Coupling between integrators that make up a PRK dual-rate integrator is incorporated in 
PRK dual-rate integrators at the design stage, and no additional algorithm is necessary to account for coupling between slow and fast differential equations.

In simulating vehicles with realistic models of tires, it was found that a PRK dual-rate integrator can be faster than a corresponding single-rate Runge-Kutta integrator by a factor of 2.4 .

While only certain kinds of models have been simulated, the methodologies developed for designing PRK dual-rate integrators are broadly applicable. For example, stabilized Runge-Kutta theory has been used to design PRK dual-rate integrators in which fast integrators have large stability regions along the imaginary axis. The same methodology can be used to design PRK dual-rate integrators in which fast integrators have large stability regions along the negative real axis.

\section{APPENDIX A-EXAMPLE DEMONSTRATING LIMITATION ON ORDER DUE TO COUPLING}

Consider two linear ODE,

$$
\left\{\begin{array}{c}
\dot{x} \\
\dot{y}
\end{array}\right\}=\left[\begin{array}{cc}
A & B \\
C & D
\end{array}\right]\left\{\begin{array}{l}
x \\
y
\end{array}\right\}
$$

where $x$ is the slow variable and $y$ is the fast variable.

Let $X_{\mathrm{i}}$ and $Y_{\mathrm{i}}$ denote the analytical solution at time $i h$. Expanding $X_{1}$ and $Y_{1}$ about $X_{0}$ and $Y_{0}$, respectively, using Taylor Series yields

$$
\begin{aligned}
& X_{1}=X(0+h)=X(0)+h X^{\prime}(0)+\frac{h^{2}}{2} X^{\prime \prime}(0)+\frac{h^{3}}{6} X^{\prime \prime \prime}\left(\xi_{x}\right) \\
& Y_{1}=Y(0+h)=Y(0)+h Y^{\prime}(0)+\frac{h^{2}}{2} Y^{\prime \prime}(0)+\frac{h^{3}}{6} Y^{\prime \prime \prime}\left(\xi_{y}\right)
\end{aligned}
$$

where $0 \leq \xi_{x} \leq h$ and $0 \leq \xi_{y} \leq h$. The prime symbol represents differentiation with respect to time $t$.

For the ODE of Eq. (A1), Eqs. (A2) and (A3) become

$$
\begin{aligned}
X_{1}= & X_{0}+h\left(A X_{0}+B Y_{0}\right)+\frac{h^{2}}{2}\left(A^{2} X_{0}\right. \\
& \left.+A B Y_{0}+B C X_{0}+B D Y_{0}\right)+O\left(h^{3}\right) \\
Y_{1}= & Y_{0}+h\left(C X_{0}+D Y_{0}\right)+\frac{h^{2}}{2}\left(C A X_{0}\right. \\
& \left.+C B Y_{0}+D C X_{0}+D^{2} Y_{0}\right)+O\left(h^{3}\right)
\end{aligned}
$$


Consider a PRK integrator that has a local error of $O\left(h^{2}\right)$ for the slow variable and a local error of $O\left(h^{3}\right)$ for the fast variable. Let $x_{\mathrm{i}}$ and $y_{\mathrm{i}}$ denote the numerical solution at time $i h$. Let the initial conditions be $x_{0}$ and $y_{0}$. It is assumed that there is no error in initial conditions; i.e., $x_{0}=X_{0}$ and $y_{0}=Y_{0}$. Let the first step in the numerical solution be

$$
\begin{aligned}
& x_{1}=x_{0}+h\left(A x_{0}+B y_{0}\right) \\
& y_{1}=y_{0}+h\left(C x_{0}+D y_{0}\right)+\frac{h^{2}}{2}\left(C A x_{0}+C B y_{0}+D C x_{0}+D^{2} y_{0}\right)
\end{aligned}
$$

Subtracting Eqs. (A6) and (A7) from Eqs. (A4) and (A5), respectively, the error in the first step of the numerical solution is

$$
\begin{aligned}
& \operatorname{error}\left(x_{1}\right)=X_{1}-x_{1}=O\left(h^{2}\right) \\
& \operatorname{error}\left(y_{1}\right)=Y_{1}-y_{1}=O\left(h^{3}\right)
\end{aligned}
$$

The next step in numerical integration yields

$$
\begin{aligned}
& x_{2}=x_{1}+h\left(A x_{1}+B y_{1}\right) \\
& y_{2}=y_{1}+h\left(C x_{1}+D y_{1}\right)+\frac{h^{2}}{2}\left(C A x_{1}+C B y_{1}+D C x_{1}+D^{2} y_{1}\right)
\end{aligned}
$$

The analytical solutions $X_{2}$ and $Y_{2}$ at time $t=2 h$ can be found by expanding about $X_{1}$ and $Y_{1}$, respectively, using Taylor Series. Using the same approach that is used to find $X_{1}$ and $Y_{1}$ yields

$$
\begin{aligned}
X_{2}= & X_{1}+h\left(A X_{1}+B Y_{1}\right)+O\left(h^{2}\right) \\
Y_{2}= & Y_{1}+h\left(C X_{1}+D Y_{1}\right)+\frac{h^{2}}{2}\left(C A X_{1}\right. \\
& \left.+C B Y_{1}+D C X_{1}+D^{2} Y_{1}\right)+O\left(h^{3}\right)
\end{aligned}
$$

Subtracting Eqs. (A10) and (A11) from Eqs. (A12) and (A13), respectively, the error in the second step is

$$
\begin{aligned}
& \operatorname{error}\left(x_{2}\right)=X_{2}-x_{2}=O\left(h^{2}\right) \\
& \operatorname{error}\left(y_{2}\right)=Y_{2}-y_{2}=h C\left[O\left(h^{2}\right)\right]+O\left(h^{3}\right)=O\left(h^{3}\right)
\end{aligned}
$$

Equations (A14) and (A15) show that the local error in the fast variable is $O\left(h^{3}\right)$. 
In addition to truncation error, there is an error that arises due to the fact that the slow variable has an error one order less than the error of the fast variable does. If $h^{2} \tau_{x}$ and $h^{3} \tau_{y}$ are taken as truncation errors for the slow and the fast variables, respectively; i.e., the error in Eqs. (A8) and (A9), then the error in the second step is

$$
\begin{aligned}
& \operatorname{error}\left(x_{2}\right)=2 h^{2} \tau_{x}+O\left(h^{3}\right) \\
& \operatorname{error}\left(y_{2}\right)=2 h^{3} \tau_{y}+h^{3} C \tau_{x}+O\left(h^{4}\right)
\end{aligned}
$$

Similar to Eqs. (A16) and (A17), errors in the third and subsequent steps can be found. In the $n$th step,

$$
\begin{aligned}
& \operatorname{error}\left(x_{n}\right)=n h^{2} \tau_{x}+O\left(h^{3}\right) \\
& \operatorname{error}\left(y_{n}\right)=n h^{3} \tau_{y}+\frac{n(n-1)}{2} h^{3} C \tau_{x}+O\left(h^{4}\right)
\end{aligned}
$$

Assuming that $n h<K$, where $K$ is a constant, the global error can be obtained by replacing $n$ by $K / h$ in Eqs. (A18) and (A19), so

$$
\begin{aligned}
& \text { global error }(x)=O(h) \\
& \text { global error }(y)=O(h)
\end{aligned}
$$

Thus, in PRK dual-rate integration, the order of integration is limited by the maximum possible order of the Runge-Kutta integrator with the smallest number of stages, which in turn in limited by the number of stages of the slow variable Runge-Kutta integrator.

\section{APPENDIX B-PLANAR RIGID RING TIRE MODEL}

The planar rigid ring tire model used here has been described by Zegelaar et al. (1993) and Zegelaar and Pacejka (1997). According to Maurice et al. (1997), planar rigid ring tire models are used to study power hop vibrations, dynamic tire responses on uneven roads, and Antilock Braking System (ABS) operation. This model is depicted in Fig. B1. The tire tread band is represented by a rigid ring. There is also a wheel hub body for each wheel, which is attached to the chassis by a revolute joint. Forces are transmitted from the rigid ring to the wheel hub by springs and dampers. Residual stiffnesses are used to represent contact patch compliance. Variables used in the model are defined below. 


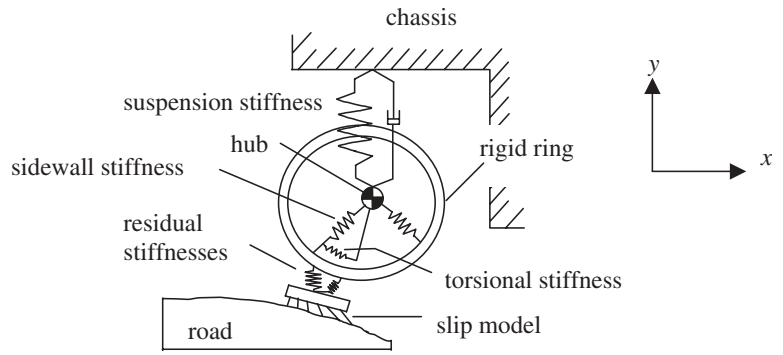

Figure B1. Planar rigid ring tire model.

External forces and torques acting on the wheel hub body can be found using well-known techniques for constructing equations of motion, such as free body diagrams:

$$
\begin{aligned}
F_{H}^{x}= & K^{x}\left(x_{R}-x_{H}\right)+C^{x}\left(\dot{x}_{R}-\dot{x}_{H}\right) \\
F_{H}^{y}= & K^{y}\left(y_{R}-y_{H}\right)+C^{y}\left(\dot{y}_{R}-\dot{y}_{H}\right)+K^{s}\left(y_{c h}-y_{H}\right) \\
& +C^{s}\left(\dot{y}_{c h}-\dot{y}_{H}\right)-m_{H} g \\
T_{H}^{\theta}= & K^{\theta}\left(\theta_{R}-\theta_{H}\right)+C^{\theta}\left(\dot{\theta}_{R}-\dot{\theta}_{H}\right)+T_{\mathrm{ext}}
\end{aligned}
$$

where $K^{x}$ and $C^{x}$ represent sidewall stiffness and damping, respectively, in the $x$-direction; $K^{y}$ and $C^{y}$ represent sidewall stiffness and damping, respectively, in the $y$-direction; $K^{\theta}$ and $C^{\theta}$ represent sidewall torsional stiffness and damping, respectively; $K^{s}$ and $C^{s}$ are the suspension stiffness and damping, respectively; $\left(x_{R}, y_{R}, \theta_{R}\right)$ and $\left(\dot{x}_{R}, \dot{y}_{R}, \dot{\theta}_{R}\right)$ represent the position and orientation and corresponding velocities, respectively, of the rigid ring; $\left(\bar{x}_{H}, \bar{y}_{H}, \bar{\theta}_{H}\right)$ and $\left(\dot{x}_{H}, \dot{y}_{H}, \dot{\theta}_{H}\right)$ represent the position and orientation and the corresponding velocities, respectively, of the wheel hub; $\left(x_{c h}, y_{c h}\right)$ and $\left(\dot{x}_{c h}, \dot{y}_{c h}\right)$ represent the position and velocity, respectively, of the point on the chassis at which the suspension connecting the chassis to wheel is attached; $m_{H}$ is the mass of the wheel hub; $g$ is acceleration due to gravity; and $T_{\text {ext }}$ is external torque, such as engine and the brake torques that are applied to the wheels.

The normal force acting at the tire-road interface, for each tire, is (Zegelaar and Pacejka, 1997)

$$
F_{N}=-K^{\text {res }}\left(y_{R}-y_{\text {road }}-y_{\text {road_0 }}\right)-C^{\text {res }} \dot{y}_{R}
$$

where $K^{\text {res }}$ and $C^{\text {res }}$ are vertical residual (tread) stiffness and damping, respectively; $y_{\text {road }}$ is road height; and $y_{\text {road_o }}$ is the relaxed spring distance between the road and the rigid ring center. 
Let

$$
\beta=\frac{2}{3} \frac{c_{p x} a^{2}}{\mu F_{N}}
$$

where $c_{p x}$ is the longitudinal tread stiffness, $a$ is the static relaxation length, and $\mu$ is the friction coefficient. The longitudinal force at the tireroad interface is then given by (Zegelaar and Pacejka, 1997)

$$
\begin{aligned}
& F_{\text {road }}=\mu F_{N}\left[3|\beta s|-3|\beta s|^{2}+|\beta s|^{3}\right] \operatorname{sign}(s), \text { for }|s| \leq \frac{1}{\beta} \\
& F_{\text {road }}=\mu F_{N} \operatorname{sign}(s), \text { for }|s|>\frac{1}{\beta}
\end{aligned}
$$

where $s$ is the longitudinal tire slip. The longitudinal force acting at the rigid ring center is thus

$$
F_{R}^{x}=F_{\text {road }}-K^{x}\left(x_{R}-x_{H}\right)-C^{x}\left(\dot{x}_{R}-\dot{x}_{H}\right)
$$

and the vertical force at each rigid ring center is given by

$$
F_{R}^{y}=F_{N}-K^{y}\left(y_{R}-y_{H}\right)-C^{y}\left(\dot{y}_{R}-\dot{y}_{H}\right)-m_{R} g
$$

where $m_{R}$ is the mass of the rigid ring.

The torque at each rigid ring center is given by

$$
\begin{aligned}
T_{R}^{\theta}= & R F_{\text {road }}+c_{r r} F_{N} \operatorname{sign}\left(\dot{x}_{R}\right)-K^{\theta}\left(\theta_{R}-\theta_{H}\right) \\
& -C^{\theta}\left(\dot{\theta}_{R}-\dot{\theta}_{H}\right)
\end{aligned}
$$

where $R$ is the radius of the rigid ring and $c_{r r}$ is the rotational rolling resistance of the tire.

The differential equation for tire slip is (Zegelaar and Pacejka, 1997)

$$
\dot{s}=\frac{\left|\dot{x}_{R}+R \dot{\theta}_{R}\right| \operatorname{sign}\left(\dot{x}_{R}\right)}{\sigma}-\frac{\left|\dot{x}_{R}\right|}{\sigma} s
$$

where the relaxation length $\sigma$ is

$$
\sigma=\frac{C_{\kappa}}{2 a c_{p x}}
$$

and $C_{\kappa}$ is the partial derivative of the longitudinal force on the rigid ring with respect to slip $s$,

$$
C_{\kappa}=\frac{\partial F_{R}^{x}}{\partial s}
$$




\section{ACKNOWLEDGMENTS}

Grateful acknowledgments are due to Dr. Dario Solis of the National Advanced Driving Simulator and Simulation Center at the University of Iowa for many useful discussions. This material is partly based upon work supported by the National Science Foundation under Grant No. 9983708.

\section{REFERENCES}

Andrus, J. F. (1979). Numerical solution of systems of ordinary differential equations separated into subsystems. SIAM Journal of Numerical Analysis 16(4):605-611.

Belytschko, T., Lu, Y. Y. (1993). Convergence and stability analyses of multi-time step algorithm for parabolic systems. Computer Methods in Applied Mechanics and Engineering 102:179-198.

Belytschko, T., Yen, H. J., Mullen, R. (1979). Mixed methods for time integration. Computer Methods in Applied Mechanics and Engineering 117/18:259-275.

Buzdugan, L. I. (1999). Multirate Integration Algorithms for Real-Time Simulation of Mechanical Systems with Interacting Subsystems. Ph.D. Thesis, Department of Mechanical Engineering, The University of Iowa.

Enright, W. H., Kamel, M. S. (1979). Automatic partitioning of stiff systems and exploiting the resulting structure. ACM Transactions on Mathematical Software 5(4):374-385.

Gear, C. W. (1980). Automatic multirate methods for ordinary differential equations. In: Lavington, S.H., ed. Information Processing 80. Amsterdam: North Holland Publishing Company, 717-722.

Gear, C. W. (1984). The numerical solution of problems which may have high frequency components. In: Haug, E.J., ed. Computer Aided Analysis and Optimization of Mechanical System Dynamics. NATO ASI Series, Vol. F9. Berlin: Springer Verlag, 335-349.

Gear, C. W., Saad, Y. (1983). Iterative solution of linear equations in ODE codes. SIAM Journal of Scientific and Statistical Computing 4(4):583-601.

Gear, C. W., Wells, D. R. (1984). Multirate Linear Multistep Methods. BIT 24 484-502.

Gunther, M., Rentrop, P. (1994). Partitioning and multirate strategies in latent electric circuits. In: Bank, R.E., Bulirsch, R., Gajewski, H., Merten, K., eds. Mathematical Modelling and Simulation of Electrical Circuits and Semiconductor Devices. Basel: Birkhauser Verlag, 33-60. 
Hairer, E., Wanner, G. (1996). Solving Ordinary Differential Equations II. Stiff and Differential-Algebraic Problems. Berlin: Springer Verlag.

Hairer, E., Norsett, S. P., Wanner, G. (1993). Solving Ordinary Differential Equations I. Nonstiff Problems. Berlin: Springer Verlag.

Hofer, E. (1976). A partially implicit method for large stiff systems of ODEs with only few equations introducing small time-constants. SIAM Journal of Numerical Analysis 13(5):645-663.

Kinnmark, I. P. E., Gray, W. G. (1984a). One step integration methods with maximum stability regions. Mathematics and Computers in Simulation 24:87-92.

Kinnmark, I. P. E., Gray, W. G. (1984b). One step integration methods of third-fourth order accuracy with large hyperbolic stability limits. Mathematics and Computers in Simulation 24:181-188.

Maurice, J. P., Zegelaar, P. W. A., Pacejka, H. B. (1998). The influence of belt dynamics on cornering and braking properties of tyres. Proceedings of the 15th IAVSD Symposium. Budapest, Hungary.

Palusinski, O. A. (1986). Simulation of dynamic systems using multirate integration techniques. Transactions of the Society for Computer Simulation 2(4):257-273.

Rentrop, P. (1985). Partitioned Runge-Kutta methods with stiffness detection and stepsize control. Numerische Mathematik 47:545-564.

Serban, R., Negrut, D., Haug, E. J. (1998). HMMWV Multibody Models, Technical Report R-211, Center for Computer Aided Design, The University of Iowa.

Solis, D. (1996). Multirate Integration Methods for Constrained Mechanical Systems with Interacting Subsystems. Ph.D. Thesis, Department of Mechanical Engineering, The University of Iowa.

Sommeijer, B. P., Shampine, L. F., Verwer, J. G. (1998). RKC: an explicit solver for parabolic PDEs. Journal of Computational and Applied Mathematics 88(2):315-326.

Strehmel, K., Weiner, R. (1984). Partitioned adaptive Runge-Kutta methods and their stability. Numerische Mathematik 45:283-300.

van der Houwen, P. (1977). Construction of Integration Formulas for Initial Value Problems. Amsterdam: North-Holland, 89-100.

Verwer, J. G. (1996). Explicit Runge-Kutta methods for parabolic partial differential equations. Applied Numerical Mathematics 22:359-379.

Weiner, R., Arnold, M., Rentrop, P., Strehmel, K. (1993). Partitioning strategies in Runge-Kutta type methods. IMA Journal of Numerical Analysis 13:303-319. 
Wells, D. R. (1982). Multirate Linear Multistep Methods for the Solution of Systems of Ordinary Differential Equations, Report UIUCDCSR-82-1093, Department of Computer Science. University of Illinois at Urbana-Champaign.

Zegelaar, P. W. A., Pacejka, H. B. (1997). Dynamic tyre responses to brake torque variations. Vehicle System Dynamics Supplement 27:65-79.

Zegelaar, P. W. A., Gong, S., Pacejka, H. B. (1993). Tyre models for the study of in-plane dynamics. Proceedings of the 13th IAVSD Symposium. Chengdu, P.R. of China.

Received November 2003

Revised March 2004

Communicated by S. Velinslay 


\section{Request Permission or Order Reprints Instantly!}

Interested in copying and sharing this article? In most cases, U.S. Copyright Law requires that you get permission from the article's rightsholder before using copyrighted content.

All information and materials found in this article, including but not limited to text, trademarks, patents, logos, graphics and images (the "Materials"), are the copyrighted works and other forms of intellectual property of Marcel Dekker, Inc., or its licensors. All rights not expressly granted are reserved.

Get permission to lawfully reproduce and distribute the Materials or order reprints quickly and painlessly. Simply click on the "Request Permission/ Order Reprints" link below and follow the instructions. Visit the U.S. Copyright Office for information on Fair Use limitations of U.S. copyright law. Please refer to The Association of American Publishers' (AAP) website for guidelines on Fair Use in the Classroom.

The Materials are for your personal use only and cannot be reformatted, reposted, resold or distributed by electronic means or otherwise without permission from Marcel Dekker, Inc. Marcel Dekker, Inc. grants you the limited right to display the Materials only on your personal computer or personal wireless device, and to copy and download single copies of such Materials provided that any copyright, trademark or other notice appearing on such Materials is also retained by, displayed, copied or downloaded as part of the Materials and is not removed or obscured, and provided you do not edit, modify, alter or enhance the Materials. Please refer to our Website User Agreement for more details.

\section{Request Permission/Order Reprints}

Reprints of this article can also be ordered at http://www.dekker.com/servlet/product/DOI/101081SME200027930 